\title{
Editorial
}

\section{Public Organizations' Crisis Preparedness}

\author{
Sanneke Kuipers $\underline{\mathrm{Q1}}$
}

The title Risk, Hazards and Crisis in Public Policy implies that the range of topics covered in this journal is rather broad, though not unlimited. In fact, the majority of our articles focuses on exactly the link between these nouns: crisis preparedness related to identified risks and hazards, as evident from public policy (both on paper and in operation). A survey of RHCPP volumes showed that preparedness and (community) resilience were the topics most often addressed in our articles of the past nine years - together good for $46 \%$ (see Kuipers \& Welsh, 2017).

But how to prepare for unknown risks, or detect anomalies in complex organizations that may turn into negative surprises: unanticipated crises for the organization(s) at hand? In this volume of RHCPP we proudly present an invited essay by Todd R. La Porte (2018), "Preparing for Anomalies, Revealing the Invisible: Public Organization Puzzles". A teacher at heart, and a lifelong top scholar on organizational reliability, Laforte takes us through the trying conditions that contemporary public organizations face, through the surprises that are likely to result from these conditions at different levels of organization, to the implications for 'anomaly seeking'.

La Porte presents to us the drivers of increasing social complexity and patterns of cross-currents that may impede effective mission accomplishment of public organizations, and the challenges these changes pose to leadership foresight (part I). Part II reveals how such drivers and currents result in organizational anomalies that can be discovered at the micro-, meso- and macro levels of organizations, including the compelling story of Seaman Zorch and the Foreign Object Damage (FOD) walk down on the USS Carl Vinson, one of the US navy's most advanced nuclear aircraft carriers. In part III ("Taking Up the Hunt") we learn what detection of anomalies implies for public organizations and their leaders by improving our frames of references for observation and listening, both incidentally and intentionally seeking to know organizational anomalies before 
they surprise us. Usually operators recognized existing patterns and have tacit knowledge that needs to become evident at strategic levels of the organization.

Next in line in this issue is a contribution that pertains to detecting feedback from the shop floor: Myers and Bearss (2018) 'Mandatory Public Health Emergency Preparedness: Analysis of the CMS Rule'. To ensure that hospitals and other medical facilities prepare for known risks (in terms public health emergencies), the Centers for Medicare and Medicaid Services (CMS) issued a concept-rule in 2013 laying out standards for public health emergency preparedness that these facilities have to meet in order to receive federal funding. The hundreds of comments submitted by the facilities they received in response are the empirical base of this study. The study reveals that different types of organizations (they vary in degree of specialization, size, type of care) see this preparedness rule and its implications as threatening their future capacity to uphold their effectiveness with respect to their core mission as health care facility. The CMS responded that they had appreciated the diversity of the facilities and their concerns in their final rule, and that the resulting rule would be applicable across institutions. For individual institutions it seems typically one of those external currents that impact both input (funding) and output (both emergency and routine public health care) that increase their organizational vulnerability in the way Laporte (2018) addresses.

In their article "Almost Accidentally: Discovering Assisted Coping After Disaster", Lauren Clay, Alex Greer and Jim Kendra from the Disaster Research Center in Delaware look into preparedness for mental health care after disaster and how the challenges this poses changed over 40 years by comparing a historic case (1974 tornado in Xenia, Oklahoma) with a more recent one (2013 tornado in Moore, Ohio). The Xenia case was "the first known systematic study of mental health service delivery after disaster during an ongoing response"(Clay et al., 2018, p. xx, see Dynes and Quarantelli, 1975 for the full report), the mental health delivery effort was the largest of its kind attempted in the US so far, and the case since then served as a model for future efforts (Clay et al., 2018: xx). The Clay et al study underlines how crucial it is to incorporate considerations of psychosocial crisis management in more generic crisis and disaster planning and preparedness (Dückers et al., 2017).

Clay et al. (2018) highlight how provision of mental healthcare after disaster moved from a medical perspective to a community-oriented approach. The community approach pertains to assisted coping: "problem-solving, prioritization of recovery needs, facilitating access to service, advocacy for service provision and other practical necessities" that help the majority of the affected people sufficiently while allowing for more specific needs-based counseling and psychosocial treatment for particular cases that are identified in the process (ibid: p. $x x$ ). The effectiveness and relevance of the community approach 'accidentally' discovered in the 1970s Xenia case, became evident again in the 2013 Moore case. In the first case there was no disaster response or mental health response plan in Xenia (the approach emerged against the advice of external medical health experts and seemed to meet the needs of the affected population). In the second 
case, Moore had a disaster plan, that did not address mental health aspects. The Moore disaster responders relied on the best practices that also characterized the Xenia approach, and were found similarly useful in 2013 and include still many insightful lessons for future disaster responses.

The next two articles in this issue are about trust in government responses to crises and risks. "The Role of Government Legitimacy and Trust in Managing Refugee Crises" by Demiroz and Unlu (2018) offers great insights from a qualitative case study on the reception of Syrian refugees by the Turkish authorities in the Turkish-Syrian border region Kobani. It shows how ethnic (Turkish-Kurdish) conflict and political circumstances affect refugees' perceptions and trust of the crisis response by the Turkish government, and also affect refugee integration and compliance to official policies. Compliance means that refugees see official policies as trustworthy and legitimate and that they are willing to register, accept government-provided services and cooperate with allocation decisions by authorities (non-compliance would be that refugees attempt to minimize any interaction with authorities). Trust and compliance are to an important extent mediated by the refugees' direct social networks, e.g. the local host communities of Kurdish people in Turkey. If the local Kurds that received refugees were supporting the Turkish government (the local community in Şanhurfa), the compliance among refugees was much higher than if local Kurdish hosts politically opposed Turkish authorities (the host community in Suruç). The findings from this study are highly relevant for other host countries with ethno-political, religious or racial tensions and the way they cope with refugee compliance or non-compliance to official refugee reception and integration policies.

The final contribution of this issue examines local communities' trust in government responses given the level of risk awareness and responsibility they attribute to state and local governments. Murphy, Greer, and Wu (2018) discuss in "Trusting Government to Mitigate a New Hazard: The Case of Oklahoma Earthquakes" how people see government's ability and willingness to deal with a new, technologically-induced, natural hazard. The Oklahoma earthquakes are allegedly produced through underground waste-water injection by the oil- and gas industry, an activity that citizens perceive as something government can control or regulate. Trust in government's ability to deal with the hazard is therefore based on whether citizens believe government is willing to curtail the industry. Public perception on government responsibility varies and this perception in turn affects individual hazard adjustment activities in local communities.

Crisis preparedness related to identified risks and hazards, as evident from public policy, the topic of most contributions to this journal thus to a large extent draws on trust between citizens and authorities. This brings us back to Laporte's essay which points at the importance of the "astuteness of leaders' foresight to recognize the emergence of conditions reducing their organization's collective capacity to weather the changes that would degrade the effectiveness [of their organization]" (2018: p. xx). The contributions to this issue of RHCPP point at a variety of crisis and disaster management efforts (preparing for public health 
emergencies, organizing effectively and legitimately for psychosocial response to disasters, refugee crisis response, and mitigation of human-induced natural hazards) in which complex social conditions and cross-currents affect the future response capacity of public organizations.

\section{References}

Clay, Lauren, Alex Greer, and Jim Kendra. 2018. "Almost Accidentally: Discovering Assisted Coping After Disaster." Risk Hazards and Crisis in Public Policy 9 (3): xx-xx.

Demiroz, Fatih, and Ali Unlu. 2018. "The Role of Government Legitimacy and Trust in Managing Refugee Crises." Risk Hazards and Crisis in Public Policy 9 (3): xx-xx.

Dückers, Michel, Joris Yzermans, Wouter Jong, and Arjen Boin. 2017. "Psychosocial Crisis Management: The Unexplored Intersection of Crisis Leadership and Psychosocial Support." Risk Hazards and Crisis in Public Policy 8 (2): 94-112.

Dynes, Russell, and Enrico Quarantelli. 1975. "The Delivery of Mental Health Services in the Xenia Tornado." Disaster Research Center, miscellaneous report 14, http://udspace.udel.edu/handle/ $19716 / 1270$

Kuipers, Sanneke, and Nicholas Welsh. 2017. "Taxonomy of the Crisis and Disaster Literature: Themes and Types in 34 Years of Research." Risk Hazards and Crisis in Public Policy 8 (4): 272-83.

Laf Porte, Todd R. 2018. "Preparing for Anomalies, Revealing the Invisible: Public Organization Puzzles." Risk Hazards and Crisis in Public Policy 9 (3): xx-xx.

Murphy, Haley, Alex Greer, and Hao-Che Wu. 2018. "Trusting Government to Mitigate a New Hazard: The Case of Oklahoma Earthquakes." Risk Hazards and Crisis in Public Policy 9 (3): xx-xx.

Myers, Nathan, and Anna Bearss. 2018. "Mandatory Public Health Emergency Preparedness: Analysis of the CMS Rule." Risk Hazards and Crisis in Public Policy 9 (3): xx-xx.

\section{公共机构的危机预备}

\section{Sanneke Kuipers}

《公共政策中的风险、危害和危机》(Risk, Hazards and Crisis in Public Policy, RHCPP) - - 此标题暗示本期刊的主题范围尽管有限却相当广泛。事实上, 本刊文章大部分聚焦于下列名词之间的准确关系：危机预备、已被识别的风险和危 险。这三者在公共政策中已经得以印证(不论是理论还是实践)。一项有关RHCPP期刊 的调查显示，危机预备和 (社区) 适应力曾是过去9年间本刊最常涉及的主题一一共占 46\%（见Kuipers and Welsh, 2017)。

然而, 如何为未知风险做好准备, 或者如何在复杂机构中探测异常呢? 这些异常 可能转变为 “惊吓” (negative surprises) : 机构眼前所面临的意料之外的危机。本 期很荣幸邀请了学者托德R. 拉波特 (Todd R. La O O r te) 撰写文章 为异常做准备, 揭 开看不见的一幕: 公共机构之疑惑。拉波特作为教授和终生研究机构可靠性 (organizational reliability) 的顶尖学者, 将带领大家感受当代公共机构面临的艰难状 况，感受机构的不同阶段状况可能带来的意外，进而体会 “寻求异常” (anomaly seeking) 的意义。 
拉波特介绍了社会复杂性日益增长的驱 动因素、交错趋势的模式(后者可能阻碍 公共机构任务的有效完成）、和这些变化对领导远见所构成的挑战(第一部分)。第二 部分解释了驱动因素和交错趋势如何 造成机构异常, 这些皆常在机构的微观层面、 中观层面和宏观层面都能被发现, 包括Seaman Zorch 的精彩故事和美国海军最先进 的核能航空母舰之一——卡尔 - 文森号 (USS Carl Vinson) 遭受的外来物损坏 (Foreign Object Damage, FOD)。第三部分( “开始搜寻”) 通过提高我们在观察和聆听时 借鉴的参考框架, 意图在机构异常发生前对其有所了解, 进而阐述了各种异常的探测 对公共机构和机构领导而言意味着什么。通常的情况是, 操作者既能识别出现有模 式，同时还具备策略性知识 (该知识体现在机构的策略层面)。

下一篇 和本期主题相符的文章有关于探测工作人员的反馈：作者Nathan Myers 和 Anna Bearss (2018)共同撰写了 公共卫生应急准备的强制执行：分析CMS规 定。为确保医院和其他医疗机构为公共卫生突发事件相关的已知风险做好准备, 医 疗保险与医疗补助服务中心 (Centers for Medicare and Medicaid Services, CMS) 于 2013年发布了一项规定，概述了医院和其他机构为接受联邦资金而必须达到的标准。 此文的实证研究基础则是上述机构针对该规定提出的成百上干的评论。研究结果显 示, 不同类型的机构 (以专业、机构大小、医疗类型进行划分) 认为此项规定及其意义 会威胁到前者在今后将卫生医疗作为核心任务予以开展时的能力。CMS回应时认 为, 其重视医疗机构的多样性和机构对该规定产生的顾虑; CMS同时认为, 最终的规定将 会适用于各机构。个别机构似乎就如同这些外部趋势之一, 它们能影响输入(获得资 金) 和输出 (包括突发事件和日常公共医疗)，从而增加各自机构的脆弱性，这与拉波 特 (2018) 的研究结果相同。

在 几乎是一场意外: 灾难之后发现协助处理 一文中, 作者Lauren Clay, Alex Greer 和 Jim Kendra(他们都来白特拉华州灾害研究中心)通过将一项历史案例 (1974 年齐尼亚市龙卷风袭击事件) 和一项近期案例 (2013年俄克拉何马州穆尔龙 卷风袭击 事件) 进行比较, 考察了灾后心理健康护理的准备, 以及灾后产生的挑战在四十多年 间发生了怎样的改变。齐尼亚一案是 “有关灾后持续响应期间心理健康服务传递的 首次系统性研究” (Clay et al, 2018, p. xx, 完整报告见 Dynes and Quarantelli, 1975)，当时的心理健康传递工作达到了目前美国同类型案例中的最大规模, 自那时 起, 此案便成为未来工作中的模范案例 (Clay et al, 2018; xx)。克雷等人 (Clay et a1) 的研究强调, 将心理社会危机管理合并入更加一般化的危机和灾害计划及预备 中有多么重要。

克 雷等人 (2018) 重点指出了提供灾后心理健康护理一事如何从医疗的们度转变 为一项以社区为导向的措施。这项社区措施有关如何协助处理 “问题解决、灾后恢 复需求的优先处理、服务的便利性、服务提供和其他实际需求的支持”。此协助处 理能充分帮助大多数受灾害影响人群, 同时允许更多的特定需求咨询, 并为处理过程 中发现的特定案例提供心理社会治疗 (ibid：p. xx)。齐尼亚一案中偶然发现的社区 措施之有效性和相关性, 在2013年穆尔一案中再次出现。齐尼亚一案中没有灾害回应 或心理健康回应计划 (社区措施的出现是为了反对外部医疗卫生专家的建议, 且该措 施似乎解决了受灾害影响人群的需求); 穆尔一案中有灾害计划, 但此计划并未涉及 心理健康方面。穆尔一案巾的灾害响应者依靠以齐尼亚灾后指施为特点的最佳实践, 这一实践发挥了类似的有效性, 并为今后灾害响应提供了许多深刻的教训。

接卜来的两篇文章有关于信任政府对危机和风险的响应。作者Fatih Demiroz 和 A1 i Unlu（2018）在 政府合法性和信任在难民危机管理巾产生的作用 一文中提供 了深刻见解, 他们用定性案例研究分析了土耳其政府在土耳其 - 叙利亚边界一一艾因 阿拉伯地区接收叙利亚难民一事。文章展示了民族冲突 (土耳其 - 库尔德冲突) 和政 
治情况将 如何影响难民的观念和他们对土耳.其政府在危机响应方面的信任度; 以及 如何影响难民统一和他们对官方政策的服从。服从即意味着难民将官方政策视作可 信任且合 法的政策, 并且他们愿意登记并接受政府提供的服务, 同时配合权威机构 下达的分配决策(而不服从则指难民尽可能减少与权威机构之间的互动)。信任和服 从足一项重要内容, 它由难民直接社会网络进行调和, 比如居住在土耳其的地方库尔 德东道主社区。如果接收难民的当地库尔德人支持土耳其政府 (如桑尼乌法的地方社 区)，则难民服从政策的概率要远高于社区反对政府的情况（如苏鲁奇的东道主社 区)。此文的研究结果对其他面临民族政治、宗教或种族冲突、以及处理难民对官方 难民 接收和统一政策服从与否时的东道主国家而言，具有高度相关性。

本 期最后一篇文章考察了地方社区对政府响应的信任度 (基于社区对国家政府和 地方政府的风险意识程度和责任程度)。作者Haley Murphy, Alex Greer 和 Hao - Che Wu (2018) 在 信任政府对新危害采取缓解措施：以俄克拉荷马州地震为例 一文中探 讨了人们如何看待政府在应对由技术导致的新自然灾害时的能力和意愿。俄克拉荷 马州地震据说是由于石油天然气产业将废水注 入地下后产生的, 这项活动在当地居 民看来应受到政府的控制和监管。因此，信任政府有能力处理此危害则基于居民是否 相信政府愿意对该产业采取限制措施。公众对政府能力的感知存在差异, 并且这种感 知反过来会影响地方社区中个人的灾害调整活动。

从公 共政策中可以看出，与已识别风险和危害相关的危机预备一一即本期火多 数文章的主题一一讲述的是居民和权威机构之间的信任关系。这一点又回到拉波特 的文章, 后者指出了 “领导者的机敏性” 的重要性, 这种机敏性能预测各种减少 “机 构集体能力”（即经受住一系列能降低机构效力的变化)情况的出现 (2018： p. xx)。 本期文章指出了一系列有关危机和灾害管理方面的工作 (为公共卫生突发事件做准: 备、有效并正当地组织灾后心理社会响应、难民危机响应、以及缓解由人 类引起的 自然灾害), 在这一过程中, 复杂社会情况和各种交错趋势会影响未来公共机构的响 应能力。

\section{参考文献}

Clay, Lauren, Alex Greer, and Jim Kendra. 2018. "Almost Accidentally: Discovering Assisted Coping After Disaster." Risk Hazards and Crisis in Public Policy 9 (3): xx-xx.

Demiroz, Fatih, and Ali Unlu. 2018. "The Role of Government Legitimacy and Trust in Managing "Refugee Crises." Risk Hazards and Crisis in Public Policy 9 (3): xx-xx.

Duckers, Michel, Joris Yzermans, Wouter Jong, and Arjen Boin. 2017. "Psychosocial Crisis Management: The Unexplored Intersection of Crisis Leadership and Psychosocial Support." Risk Hazards and Crisis in Public Policy 8 (2): 94-112.

Dynes, Russell, and Enrico Quarantelli. 1975. "The Delivery of Mental Health Services in the Xenia Tornado." Disaster Research Center, miscellaneous report 14, http://udspace.udel.edu/handle/ $19716 / 1270$

Kuipers, Sanneke, and Nicholas Welsh. 2017. "Taxonomy of the Crisis and Disaster Literature: Themes and Types in 34 Years of Research." Risk Hazards and Crisis in Public Policy 8 (4): 272-83.

La Porte, Todd R. 2018. "Preparing for Anomalies, Revealing the Invisible: Public Organization Puzzles." Risk Hazards and Crisis in Public Policy 9 (3): xx-xx.

Murphy, Haley, Alex Greer, and Hao-Che Wu. 2018. "Trusting Government to Mitigate a New Hazard: The Case of Oklahoma Earthquakes." Risk Hazards and Crisis in Public Policy 9 (3): xx-xx. 
Myers, Nathan, and Anna Bearss. 2018. "Mandatory Public Health Emergency Preparedness: Analysis of the CMS Rule." Risk Hazards and Crisis in Public Policykkk 9 (3): xx-xx.

la preparación para las crisis por parte de las organizaciones

\section{Sanneke Kuipers}

El título Risk, Hazards and Crisis in Public Policy sugiere que el rango de temas que se cubren en esta revista es muy amplio, a pesar de no ser ilimitado. De hecho, la mayoría de nuestros artículos está enfocada en exactamente el vínculo entre estos sustantivos: preparación para las crisis relacionadas a riesgos y peligros identificados, como son evidentes desde las políticas públicas (tanto en papel, como en la operación). Una encuesta de volúmenes de RHCCP demostró que la preparación y la resiliencia (de la comunidad) eran temas que a menudo abordábamos en nuestros artículos en los últimos nueve años - ambos buenos para el $46 \%$ (ver Kuipers y Welsh, 2017).

Pero ?cómo prepararse para los riesgos desconocidos, o detectar anomalías en organizaciones complejas que podrían convertirse en sorpresas negativas: crisis no anticipadas para las organizaciones a la mano? En este volumen de RHCCP presentamos orgullosamente un ensayo del invitado Todd R. Läorte (2018), "Preparándose para las anomalías, revelando lo invisible: los rompecabezas de la organización pública." Un profesor de corazón, y de los mejores académicos de la fiabilidad organizacional, Laporte nos lleva a través de las condiciones difíciles que las organizaciones públicas contemporáneas enfrentan, a través de las sorpresas que pueden posiblemente resultar de estas condiciones en diferentes niveles de organización, hasta las implicaciones para la 'búsqueda de anomalías.'

Laf Torte nos presenta los catalizadores de una complejidad social en crecimiento y nos expone los patrones de las corrientes cruzadas que podrían impedir el cumplimiento efectivo de la misión de las organizaciones públicas, y los retos que estos cambios representan para la previsión de los líderes (parte I.) La parte II revela qué tanto estos catalizadores y corrientes tienen un resultado en las anomalías organizacionales que pueden ser descubiertas a nivel micro, meso y macro de las organizaciones, incluyendo la convincente historia del marinero Zorch y el trayecto de daño por objetos extraños (FOD) en el USS Carl Vinson, uno de los portaaviones nucleares más avanzados de la marina de los EE. UU. En la parte III ("Empezando la cacería") aprendemos qué implicaciones tiene la detección de anomalías para las organizaciones públicas y para sus líderes al mejorar sus marcos de referencia para la observación y la escucha, tanto incidentalmente como internacionalmente, buscando identificar las anomalías organizacionales antes de que nos sorprendan. Usualmente los operadores reconocieron los patrones existentes y tienen un conocimiento tácito que tiene que volverse evidente en los niveles estratégicos de la organización. 
A continuación en esta edición hay una contribución que tiene que ver con detectar retroalimentación desde el primer nivel: Nathan Myers y Anna Bearss (2018) 'Preparación obligatoria para las emergencias de salud pública: análisis de la regla CMS.' Para asegurar que los hospitales y las otras instalaciones médicas se preparan para los riesgos conocidos (en términos de las emergencias de salud pública), los Centros para Cuidados y Servicios Médicos (CMS) emitieron una regla de concepto en 2013 detallando estándares para la preparación para emergencias de salud pública que estas instalaciones tienen que cumplir para poder recibir fondos federales. Los cientos de comentarios emitidos por las instalaciones que recibieron en respuesta son la base empírica de este estudio. El estudio revela que los diferentes tipos de organizaciones (varían en grado de especialización, tamaño y tipo de cuidado) ven esta regla de preparación y sus implicaciones como una amenaza para su futura capacidad de sostener su efectividad respecto a su misión principal como una instalación de salud. El CMS respondió que apreciaban la diversidad de las instalaciones y sus preocupaciones en su regla final y que la regla que resultó de esto se aplicaría en todas las instituciones. Para las instituciones individuales parece típicamente una de esas corrientes externas que impactan tanto la financiación, como los servicios que se prestan (el cuidado de emergencia y de rutina) que incrementan su vulnerabilidad organizacional de la forma en que LadPorte (2018) explica.

En el artículo "Casi accidentalmente: descubriendo el lidiar asistido después de los desastres," Lauren Clay, Alex Greer y Jim Kendra del Disaster Research Center en Delaware examinan la preparación para desastres de la salud mental y cómo los desafíos resultantes han cambiado más de 40 años al comparar un caso histórico (el tornado Xenia en Oklahoma en 1974) con uno más reciente (tornado en Moore en Ohio en 2013). El caso Xenia fue "el primer estudio sistemático conocido de esfuerzos de prestación de servicios de la salud mental después de los desastres durante una respuesta en curso"(Clay et al, 2018, p.xx, ver Dynes y Quarantello, 1975 para el reporte completo), este esfuerzo de prestación de servicios de salud mental fue el más significativo de este tipo en los EE. UU. hasta ahora, y el caso desde ese entonces ha servido como un modelo para esfuerzos futuros (Clay et al, 2018: xx). El estudio de Clay et al resalta lo crucial que es incorporar las consideraciones de la gestión de crisis psicosociales en crisis más genéricas y en la planeación y preparación de desastres (Dǘckers et al, 2017).

Clay et al (201) resalta cómo la provisión de la salud mental después de los desastres se trasladó de una perspectiva médica a un acercamiento orientado hacia la comunidad. El acercamiento orientado hacia la comunidad tiene que ver con el lidiar asistido: "resolución de problemas, priorización de necesidades de recuperación, facilitar el acceso al servicio, defensa a favor de la provisión de servicios y otras necesidades prácticas" que ayudan a la mayoría de la gente afectada suficientemente mientras permiten una consejería más basada en necesidades y tratamiento psicológico para casos particulares que están identificados en el proceso (ibid: p.xx). La efectividad y relevancia del acercamiento de la comunidad que fue 'accidentalmente' descubierta en el caso Xenia de los 1970s se volvió evidente de nuevo en el caso Moore de 2013. En el primer caso no había 
respuestas a desastres o plan respuesta de salud mental en Xenia (el acercamiento surgió a pesar del consejo de expertos de salud externos y parecía satisfacer las necesidades de la población afectada). En el segundo caso, Moore tenía un plan de desastres, que no abordó los aspectos de salud mental. Los que respondieron al desastre Moore se apoyaron en las mejores prácticas que también caracterizaron al acercamiento de Xenia, y fueron también útiles en 2013 e incluyen todavía muchas lecciones reveladoras para el futuro de las respuestas a desastres.

Los próximos dos artículos en esta edición son acerca de la confianza en las respuestas del gobierno a las crisis y riesgos. "El papel de la legitimidad del gobierno y la confianza al gestionar crisis de refugiados" por Fatih Demiroz y Ali Unlu (2018) ofrece mucha información de un caso cuantitativo acerca de la acogida de refugiados sirios por parte de las autoridades turcas en la región limítrofe entre Siria y Turquía llamada Kobani. Muestra cómo el conflicto étnico entre turcos y kurdos y las circunstancias políticas afectan las percepciones de los refugiados y la confianza de la respuesta a la crisis por parte del gobierno turco, y también cómo afectan la integración de refugiados y el cumplimiento de las políticas oficiales. Cumplimiento quiere decir que los refugiados ven las políticas oficiales como algo legítimo y digno de confianza y están dispuestos a registrarse y aceptar servicios proporcionados por el gobierno y cooperar con la asignación de decisiones por parte de las autoridades (el no cumplimiento sería que los refugiados intentaran minimizar cualquier interacción con las autoridades). La confianza y el cumplimiento son en gran parte mediados por las redes sociales directas de los refugiados, por ejemplo, las comunidades anfitrionas locales de kurdos en Turquía. Si los kurdos locales que recibieron refugiados estaban apoyando al gobierno turco (la comunidad local en Şanhurfa), el cumplimiento entre los refugiados era mucho más alto que si los anfitriones locales kurdos estuvieran políticamente opuestos a las autoridades turcas (la comunidad anfitriona en Suruç). Los hallazgos de este estudio fueron altamente relevantes para otras comunidades anfitrionas con tensiones etnopolíticas, religiosas o raciales y la forma en que lidian con el cumplimiento o nocumplimiento de los refugiados con la recepción oficial de refugiados y políticas de integración.

La contribución final a esta edición examina la confianza de las comunidades locales en las respuestas del gobierno dado el nivel de consciencia del riesgo y la responsabilidad que le atribuyen a los gobiernos locales y estatales. Haley Murphy, Alex Greer y Hao-Che Wu (2018) discuten en "Confiando en el gobierno para mitigar un nuevo peligro: el caso de los terremotos de Oklahoma" cómo la gente ve la habilidad del gobierno y su deseo de lidiar con un peligro natural nuevo que ha sido causado por la tecnología. Los terremotos de Oklahoma fueron, al parecer, causados por la inyección de agua residual por la industria del petróleo, una actividad que los ciudadanos perciben como algo que el gobierno puede controlar o regular. La confianza en la habilidad del gobierno para lidiar con el peligro está entonces basada en la posibilidad de que los ciudadanos crean que el gobierno está dispuesto a ponerle obstáculos a la industria. La percepción pública de la responsabilidad del gobierno varía y esta percepción a su vez afecta las actividades de ajuste a peligros individuales en las comunidades locales. 
La preparación en casos de crisis relacionada a riesgos y peligros identificados, como evidenciada a través de las políticas públicas, es el tema de muchas contribuciones a esta revista y se basa en la confianza entre los ciudadanos y las autoridades. Esto nos trae de vuelta al ensayo de La Porte que resalta la importancia de la "astucia" de la previsión de los líderes para reconocer el surgimiento de las condiciones que reducen la capacidad colectiva de la organización para lidiar con los cambios que podrían degradar la efectividad [de su organización]" (2018: p. xx). Las contribuciones a esta edición de RHCCP apuntan a una variedad de crisis y esfuerzos de gestión de desastres (preparación para emergencias de salud pública, organizarse efectiva y legítimamente para la respuesta psicológica a desastres, respuesta a las crisis de refugiados y mitigación de peligros naturales causados por humanos) en los que condiciones sociales complejas y corrientes cruzadas afectan la respuesta futura a la capacidad de las organizaciones públicas.

\section{Referencias}

Clay, Lauren, Alex Greer, and Jim Kendra. 2018. "Almost Accidentally: Discovering Assisted Coping After Disaster." Risk Hazards and Crisis in Public Policy 9 (3): xx-xx.

Demiroz, Fatih, and Ali Unlu. 2018. "The Role of Government Legitimacy and Trust in Managing Refugee Crises." Risk Hazards and Crisis in Public Policy 9 (3): xx-xx.

Duckers, Michel, Joris Yzermans, Wouter Jong, and Arjen Boin. 2017. "Psychosocial Crisis Management: The Unexplored Intersection of Crisis Leadership and Psychosocial Support." Risk Hazards and Crisis in Public Policy 8 (2): 94-112.

Dynes, Russell, and Enrico Quarantelli. 1975. "The Delivery of Mental Health Services in the Xenia Tornado." Disaster Research Center, miscellaneous report 14, http://udspace.udel.edu/handle/ $19716 / 1270$

Kuipers, Sanneke, and Nicholas Welsh. 2017. "Taxonomy of the Crisis and Disaster Literature: Themes and Types in 34 Years of Research." Risk Hazards and Crisis in Public Policy 8 (4): 272-83.

La Porte, Todd R. 2018. "Preparing for Anomalies, Revealing the Invisible: Public Organization Puzzles." Risk Hazards and Crisis in Public Policy 9 (3): xx-xx.

Murphy, Haley, Alex Greer, and Hao-Che Wu. 2018. "Trusting Government to Mitigate a New Hazard: The Case of Oklahoma Earthquakes." Risk Hazards and Crisis in Public Policy 9 (3): xx-xx.

Myers, Nathan, and Anna Bearss. 2018. "Mandatory Public Health Emergency Preparedness: Analysis of the CMS Rule." Risk Hazards and Crisis in Public Policy 9 (3): xx-xx. 